Einführung zum Thema: NGS aktuell

medgen $2019 \cdot 31: 185-190$

https://doi.org/10.1007/s11825-019-0240-8

(C) Springer Medizin Verlag GmbH, ein Teil von Springer Nature 2019

\section{Jahre NGS in der \\ Medizin - Rückblick und Standortbestimmung}

\section{NGS in der Forschung}

Wie im Falle der Array-CGH-Technik, kamen die unter next-generation sequencing (NGS) zusammengefassten Verfahren der Hochdurchsatz-DNA-Sequenzierung zunächst in der Forschung zum Einsatz - insbesondere mit dem Ziel der Identifizierung neuer Gene für monogene Erkrankungen - bevor sie eine technische Reife erreicht hatten, die ab etwa 2010 eine zunehmende Anwendung in der molekulargenetischen Routinediagnostik erlaubte.

Einige Studien markieren Meilensteine, die schon früh eindrucksvoll das Potential von NGS demonstrierten:

Zunächst wurde die neue Möglichkeit der simultanen Anreicherung und Sequenzierung oft noch auf Gene (bzw. i.d.R. ihre Exons) zuvor kartierter chromosomaler Kandidatenregionen angewendet [1-3]. Solche jeweils maßgeschneiderten Ansätze, die immer projektspezifisch konfiguriert werden mussten, wurden schnell verlassen zugunsten umfassenderer Alternativen, bei denen auf Standardprodukte der Anbieter zurückgegriffen werden kann die Anreicherung/Sequenzierung des gesamten Exoms (whole-exome sequencing, WES) oder Genoms (whole-genome sequencing, WGS).

Durch den Vergleich der WES-Daten mehrerer nicht-verwandter Patienten mit Freeman-Sheldon-Syndrom konnte das bekannte Krankheitsgen, MYH3, zuverlässig identifiziert werden [4]. Eine entsprechende Herangehensweise bei Patienten mit dem sporadisch auftreten-

\author{
Hanno J. Bolz · Alexander Hoischen ${ }^{2}$ \\ 'Senckenberg Zentrum für Humangenetik, Frankfurt am Main, Deutschland \\ ${ }^{2}$ Department of Human Genetics \& Department of Internal Medicine, Radboud Institute of Medical Life \\ Sciences, Radboud University Medical Center, Nijmegen, Niederlande
}

\title{
NGS: Gestern, heute und morgen
}

den Schinzel-Giedion-Syndrom führte zur Identifikation heterozygoter $\mathrm{Mu}$ tationen im Gen SETBP1 [5] - dem ersten mittels WES gefundenen Gen für eine autosomal-dominant vererbte Erkrankung. Die Erkenntnis, dass es sich um Neumutationen der elterlichen Keimbahn handelte, trug maßgeblich dazu bei, dass man nachfolgend weitere mutmaßlich auf Neumutationen beruhende Erkrankungen nicht nur durch vergleichendes WES mehrerer Patienten, sondern vor allem durch den Abgleich ihrer WES-Datensätze mit denen der Eltern aufklärte [6-8]. Da diese TrioWES-Analysen nicht nur Neumutationen in Exons aufdecken, sondern bei autosomal-rezessiven Mutationen eine Segregationsanalyse bzw. sogar Haplotypisierung gestattet, ist ihre Anwendung insbesondere für sporadische Erkrankungsfälle und bei sinkenden Kosten immer attraktiver geworden.

Zunehmend wurde auch die WGSAnalyse für die Krankheitsgenidentifizierung herangezogen $[9,10]$. Mehrere Studien zeigten, dass WGS hinsichtlich exonischer Sequenzen verlässlichere Ergebnisse liefert als WES $[11,12]$.

Darüber hinaus haben sich die NGSTechniken und -Plattformen stetig weiterentwickelt (• Abb. 1).

Die Zahl der in einem Jahr neu identifizierten Krankheitsgene wuchs mit dem Anteil, den NGS (vor allem WES und WGS) daran hatte. Seit dem Höchststand im Jahr 2012 sinkt diese Zahl wieder und liegt aktuell etwa auf dem Niveau der PräNGS-Ära, 2003 ([19]; • Abb. 2).

Dieses Phänomen ist sicherlich hauptsächlich dadurch begründet, dass die meisten ursächlichen Gene für viele genetisch heterogene Erkrankungen jetzt bekannt sind (dies zeigen Abklärungs- quoten bei NGS-Panel-Diagnostiken von oft $70 \%$, bei einigen Phänotypen sogar mehr). Patienten bzw. Familien, deren Erkrankungen auf selten am Phänotyp beteiligte, noch unbekannte Gene zurückgehen, sind mittlerweile entsprechend schwer zu finden.

\section{NGS in der Diagnostik}

Die Anwendung von NGS in der molekulargenetischen Diagnostik hat die $\mathrm{Hu}$ mangenetik, die lange ein Nischendasein führte, ins Zentrum der Medizin gerückt. Praktisch alle Verdachtsdiagnosen mit einer vermuteten genetischen Grundlage sind jetzt untersuchbar geworden. Je nach Indikation bzw. der jeweiligen genetischen Heterogenität [20] werden meist Untersuchungen von Gen-Panels, WES oder auch WGS durchgeführt. Dabei gelingt das Auffinden kausaler Varianten sowohl diagnostisch als auch bei wissenschaftlichen NGS-Analysen bei einem signifikanten Teil der Patienten nicht.

Dieses Themenheft behandelt die Gründe für dieses Problem und erläutert viel versprechende Ansätze für dessen Lösung.

\section{Im toten Winkel: Versteckte Krankheits- ursachen}

Mutationen im nicht-kodierenden Bereich: Weil die ursächlichen Mutationen für monogene Erkrankungen $\mathrm{zu}$ mehr als $80 \%$ in den Exons (oder den angrenzenden intronischen Bereichen/ Spleißstellen) liegen, die überdies nur etwa $1 \%$ des Genoms ausmachen, fokussieren sich die meisten NGS-Panels bis hin zu WES auf diese Bereiche. 
Konzept: Krankheitsgen-Identifizierung mittels Exom-Sequenzierung (WES)[4]
Erstes Long-Read-Genom mittels SMRT-Sequenzierung [27]
Erstes Long-Read-Genom mittels

Nanopore (ONT)-Sequenzierung [28]
Erstes individuelles Genom Kopplungsanalyse mittels WES [58] Haplotypisiertes 'Linked-Long-Read'mittels NGS sequenziert [56]
LOH-Mapping mittels WES [59]
'Linked-Read-Analyse' ermöglicht Einzelzell- Mutationsanalyse [61]

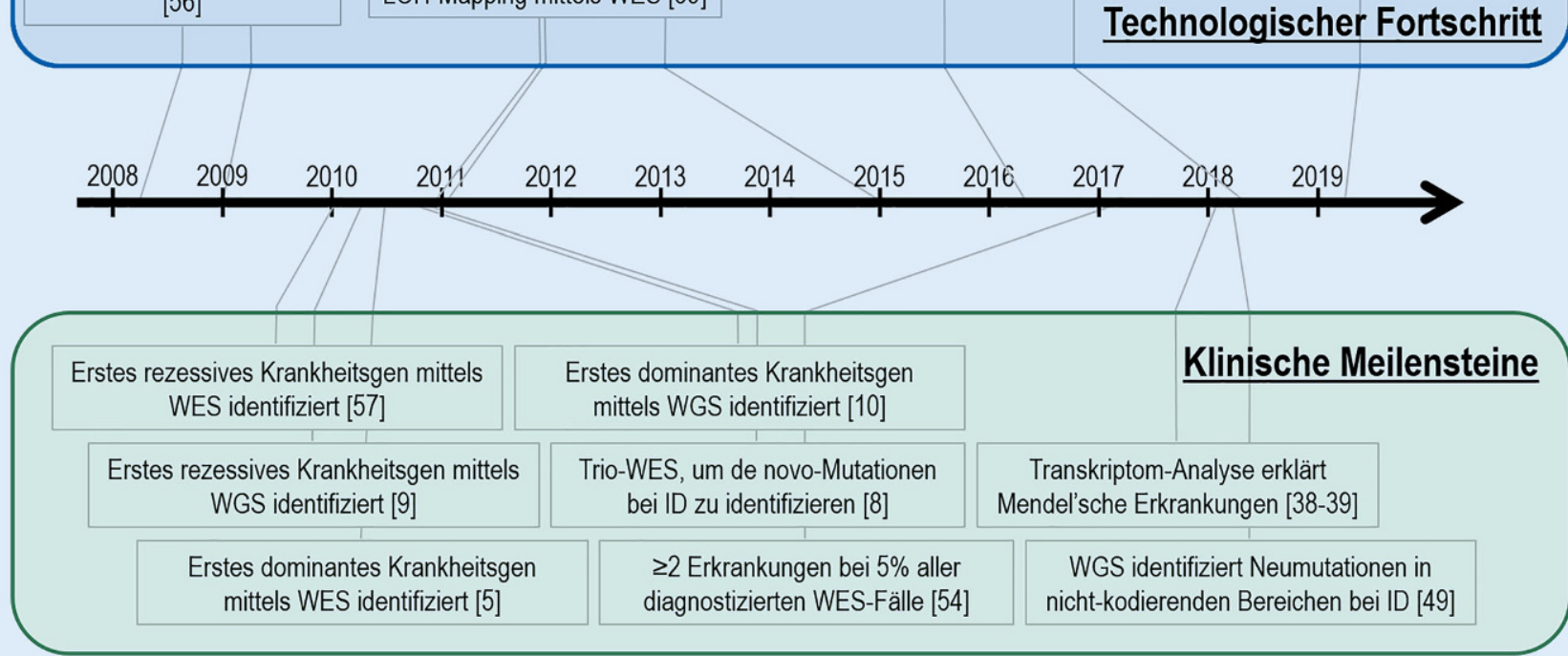

Abb. $1 \Delta$ Zeitstrahl zu technischen und klinischen Meilensteinen [13-18]

\section{Folgende Mutationstypen werden daher auf diesem Wege nicht gefunden:}

Tief-intronische Mutationen: Häufig generieren sie neue Spleißstellen, sodass in intronischer Sequenz neue Exone entstehen. Ein bekanntes Beispiel ist die häufigste Mutation bei Leberscher congenitaler Amaurose, c. $2991+1655 \mathrm{~A}>\mathrm{G}$ in CEP290 [21]. Bei Patienten mit Morbus Stargardt, einer weiteren autosomal-rezessiven Netzhautdystrophie, beruht die missing heritability von einfach heterozygoten Mutationsträgern mitunter ebenfalls auf solchen Mutationen [22, 23] vermutlich wird dies auch auf viele weitere Erkrankungen zutreffen (siehe dazu auch den Beitrag von Prokisch in diesem Heft).

Regulatorische Genregionen werden in diagnostischen Sequenzierungen meist nicht erfasst, oder erfasste Varianten werden mitunter nicht als kausal in Betracht gezogen - wie das Beispiel von Mutationen in PolyadenylierungsSignalsequenzen bei syndromaler Mikrophthalmie veranschaulicht [24]. Die
Detektion beeinträchtigter Expression ist eine Domäne der RNA-Sequenzierung (s. Artikel von Prokisch in diesem Heft), ebenso kann der Nachweis einer zu geringen Proteinmenge mittels Proteomics erbracht werden (siehe den Beitrag von Prokisch in diesem Heft).

\section{Lange nicht-kodierende RNAs}

(lncRNAs) werden vor allem im Gehirn exprimiert, wo sie wiederum die Expression zahlreicher Gene während der Hirnentwicklung regulieren. Mittlerweile wurden ursächliche Mutationen in mehreren IncRNAs bei Patienten mit Autismus und mentaler Retardierung nachgewiesen - ein Hinweis, dass zumindest lncRNAs mit zum jeweiligen Phänotyp „passenden“ Expressionsmustern bei der Mutationssuche berücksichtigt werden sollten $[25,26]$.

Strukturelle Mutationen: Der Schritt von WES zu WGS als diagnostische Routine-Technologie zeichnet sich bereits ab [12]. Auch bei nahezu kompletter Abdeckung des Genoms können jedoch mit Short-Read-Technologien (z. B. Illumina) nicht alle Varianten identifiziert werden. Dies gilt besonders für strukturelle Varianten (SVs; Insertionen/ Deletionen) und Repeat-Expansionen. Hier bieten neue Long-Read-Sequenzierverfahren wie Nanopore- (ONT), SMRT- (PacBio) und Long-Read-Optical-Mapping Technologien (z.B. Bionano) vielversprechende Ansätze. So wies das erste mit SMRT-Sequenzierung analysierte menschliche Genom mehr SVs auf als die erste Phase des 1000-Genom-Projekts [27], und auch erste Nanopore-basierte Sequenzierungen des menschlichen Genoms liegen nun vor [28]. Diese Technologien könnten eine neue Ära der Genomanalyse einläuten, weil bei neu assemblierten personalisierten Genomen die Abhängigkeit von Referenz-Genomen entfällt. "Perfekte Genom-Analysen“" mit vollständiger Identifizierung aller Varianten könnten bald als generischer Genomtest zur Verfügung stehen. Der aktuelle Stand und die Anwendungen von Long-ReadSequenzierungen werden von Kraft und Kurth in diesem Heft diskutiert.

Vererbbare DNA- und ChromatinMethylierungsmuster erklären vermutlich einige ungeklärte Erkrankungen. 


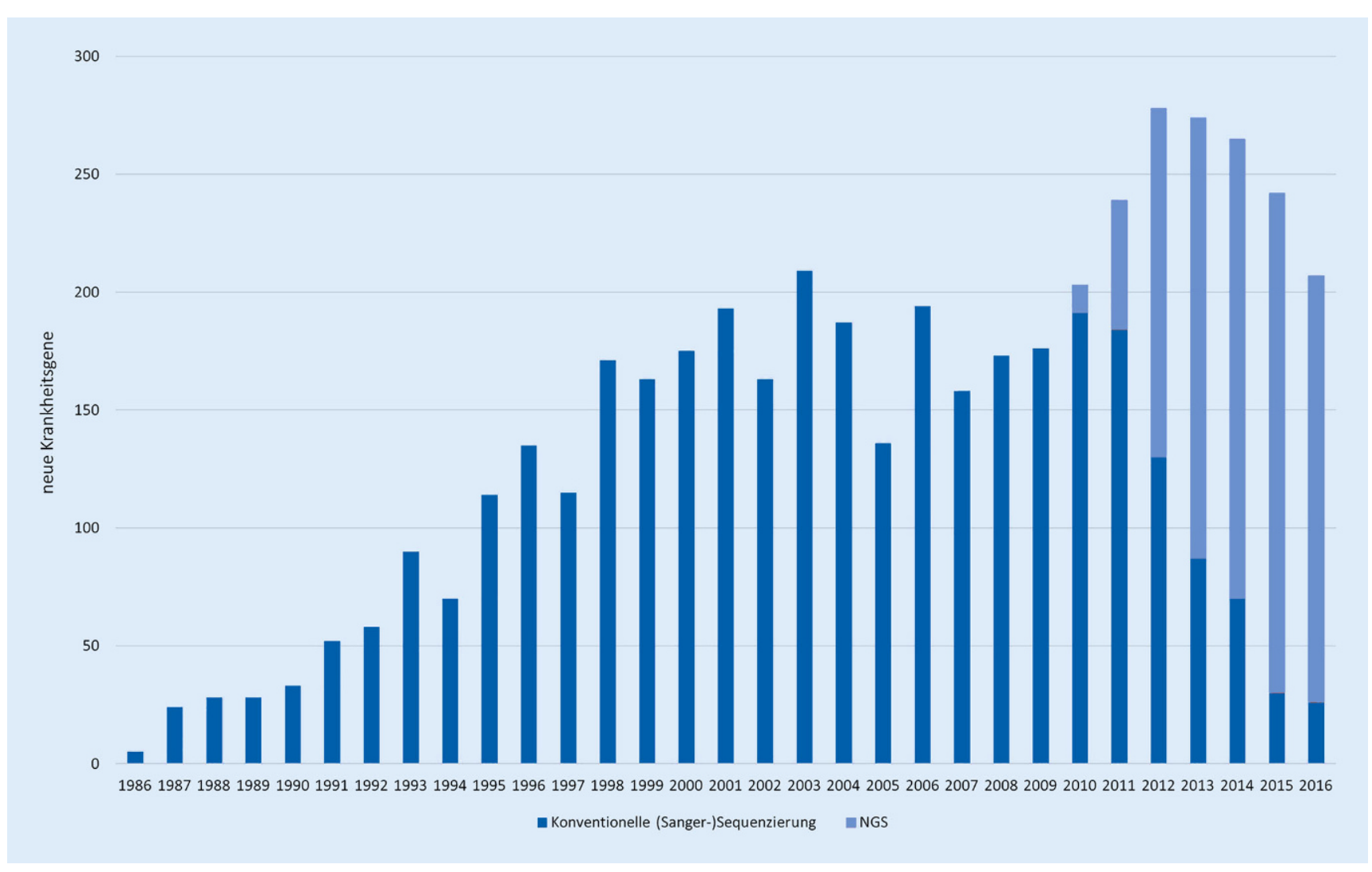

Abb. 2 ム Jährlich neu identifizierte Krankheitsgene und verwendete Sequenziertechniken. (Modifiziert nach [19])

So konnten Methylierungsdefekte als Ursache familiärer Krebserkrankungen, bei denen Sequenzen bekannter prädisponierender Gene keine Auffälligkeiten aufwiesen, identifiziert werden [29]. Diese und andere Epimutationen werden im Übersichtsartikel von Zeschnigk und Horsthemke behandelt.

Mosaike: Nicht nur lokal ausgeprägte Erkrankungen wie das Proteus-Syndrom können aufpost-zygotischen Mutationen beruhen: Etwa 5\% der Autismuserkrankungen, die i.d. R. auf Neumutationen in der elterlichen Keimbahn beruhen, resultieren aus post-zygotischen, in somatische Mosaike einmündenden Mutationen [30].

Oligogene Vererbung: Der Anteil dioder oligogener Mechanismen, quasi als Brücke zu den multifaktoriellen Erkrankungen, ist aktuell schwer beurteilbar. Beim Bardet-Biedl-Syndrom (BBS) etwa ließ sich ein hoher Anteil oligogener Konstellationen, trotz vermeintlich beweisenden funktionellen Untersuchun- gen im Tier-(häufig Zebrafisch-)Modell nicht bestätigen [31]. Selbst multiple Heterozygotien in Genen, deren Produkte miteinander im gleichen funktionellen Kompartment, z.B. dem primären $\mathrm{Zi}$ lium, interagieren, repräsentieren oft lediglich zufällige Anlageträgerschaften, ohne dass ein krankheitsverursachendes Zusammenwirken vorliegt. Um von einer oligogenen Vererbung ausgehen zu können, sind umfangreiche genomische Abklärung (Ausschluß monogener Ursachen), Segregations- und funktionelle Analysen zu fordern. Bei der Mutation c.428delG in KIAA0586 etwa ist mittlerweile klar, dass es sich um eine hypomorphe Mutation handelt, die selbst homozygot vermutlich erst zur Erkrankung (Joubert-Syndrom, JBTS) führt, wenn Varianten in weiteren JBTSLoci hinzukommen [32, 33]. Sinkende Preise und steigende Sequenzierkapazitäten erlauben zunehmend die Analyse komplex-genetischer Erkrankungen in großen Kohorten. Dies wird helfen, den Beitrag seltener und häufiger Varianten im Spektrum von mono-, oligo- und polygenen sowie multifaktoriellen Erkrankungen besser zu verstehen. Die Rolle von NGS bei komplex-genetischen Erkrankungen, insbesondere hinsichtlich seltener Varianten bei häufigen Erkrankungen, wird im Artikel von Ludwig et al. näher beleuchtet.

\section{Erfasste, aber nicht erkannte Mutationen:}

Hypomorphe Mutationen, inkomplette Penetranz: Einer der wichtigsten Filter bei der Auswertung umfangreicher NGS-Daten ist die Frequenz in einer gesunden Referenzpopulation (minor allele frequency, MAF). Bei autosomalrezessiven Mutationen etwa erwartet man, dass sie in Datenbanken wie Ex$\mathrm{AC}$ und gnomAD allenfalls selten und nicht homozygot annotiert sind. Hypomorphe rezessive Mutationen können jedoch relativ häufig in solchen Datenbanken - und dann mitunter auch homozygot - auftauchen. Ein solches Beispiel ist die mit autosomal-rezessiver Makuladystrophie assoziierte CDHR1- 


\section{Lehren aus 10 Jahren WES}

Die systematische Nutzung insbesondere von WES bei klinisch-diagnostischen Fragestellungen hat zu wichtigen Erkenntnissen geführt, die über das

Finden des Krankheitsgene/der kausalen Mutationen hinausgehen:

1. Die Krankheitsursache bleibt bei vielen Patienten nach WES ungeklärt.

2. Neumutationen: Ein Individuum trägt im Durchschnitt 1-2 exonische Neumutationen [47]. Sie sind häufig Ursache sporadisch auftretender monogener Erkrankungen (ca. $50 \%$ bei schwerer Entwicklungsverzögerung/Autismus). WES von Eltern-Kind-Trios mit gezielter Analyse auf de novo-Ereignisse macht diese schnell identifizierbar [48]; das gilt auch für WGS-Auswertungen, bei dem dies zusätzlich die Detektion kausaler struktureller und nicht-kodierender Varianten erleichtert [49].

3. Balancierte zytogenetische (Neu-) Mutationen: Solange WGS und dessen intensive Auswertung auf strukturelle Varianten nicht diagnostische Routine sind, behalten insbesondere Karyotypisierungen bei Patienten mit geistiger Behinderung ihre Berechtigung. Balancierte reziproke Translokationen etwa fallen auch bei genomweiter Sequenzanalyse und Quantifizierung (Array-CGH) nicht auf. Handelt es sich um Neumutationen, so ist dies wie bei de novo-Punktmutationen ein starker Hinweis auf Pathogenität - und die gezielte Bruchpunktanalyse im WGS sehr effizient möglich [50-52].

4. Etwa $5 \%$ der Patienten mit V. a. eine monogene Erkrankung haben tatsächlich $\geq 2$ genetische Erkrankungen [53, 54]. Die Wahrscheinlichkeit dafür ist insbesondere bei (enger) elterlicher Konsanguinität erhöht. Vor dem NGS-Zeitalter gab oft erst die klinische "Aufspaltung" eines Syndrom-Phänotyps bei Geschwistern von Index-Patienten Anlass zu weiterer (aufwendiger) Abklärung [55].

5. Die quantitative Auswertung von Panel-NGS bzw. WES ermöglicht die Detektion von Kopienzahlvarianten (copy number variations, CNVs), vermeidet diagnostische Lücken v. a. bei Erkrankungen, die die Indikationen für eine Array-CGH nicht erfüllen und erhöht deren Abklärungsquote um bis zu $6 \%[56]$.

6. Re-Analysen der WES-Rohdaten können durch verbessertes Abrufen, Detektion und aktualisierte Annotation von Varianten, evidenzbasierte Filterstrategien und die Kenntnis neuer Krankheitsgene den diagnostischen Ertrag erhöhen [57]. Zudem kann auf Mutationen im geringgradigen Mosaik gefiltert werden, die der Standardanalyse entgehen [58].

7. Meta-Analysen ungeklärter, klinisch vergleichbarer Fälle können - analog zum wissenschaftlichen Vorgehen (s. oben [5]) - durch Nachweis kausaler Mutationen, auch in neuen Krankheitsgenen, bei mehreren Patienten zur Aufklärung führen [59].

8. Allelische Erkrankungen: Mutationen in vielen Genen sind mit z.T. völlig verschiedenen Krankheiten und Erbgängen (dominant vs. rezessiv) assoziiert. Gründe sind z. B. verschiedene Mutationstypen (z. B. Funktionsgewinn- vs. -verlust, oft abhängig von der Position im Gen) sowie die Affektion von Proteindomänen völlig unterschiedlicher Funktion (z. B. führen trunkierende DIAPH1-Mutationen überwiegend zu autosomal-rezessiver Mikrozephalie, bei C-terminaler Lage jedoch zu dominanter Schwerhörigkeit [60, 61]).

9. Mutationsdatenbanken: hilfreiche Ressourcen bei der Variantenbewertung, jedoch selbst im Falle kostenpflichtiger Angebote (z. B. HGMD) oft reich an fälschlich als pathogen annotierten Varianten oder sogar Genen, oft durch Prä-NGS-Studien. Die Flut von WES-/WGS-Daten hat das Potential einer retrospektiven Bereinigung dieser Datenbanken, aber auch das Risiko einer weiteren Kontamination mit "falschen Mutationen". Gefordert ist hier höchste Sorgfalt sowohl bei Datenbankkuratoren als auch bei NGS-Anwendern. Um Varianten aufgrund ihrer Seltenheit in einer Datenbank nicht vorschnell als pathogen zu klassifizieren, ist die Verwendung populationsspezifischer Datenbanken wichtig.

10. Borderline-Phänotypen: Das Beispiel trunkierender CUX1-Mutationen, die zu aufholbarer psychomotorischer Entwicklungsverzögerung führen, aber auch bei Gesunden mit niedrig-normaler bzw. normaler Intelligenz - einem traditionell als multifaktoriell betrachteten Phänotyp - gefunden werden [62], zeigt, dass solche Varianten in Populationsdatenbanken oder bei gesunden Verwandten des Patienten (Segregationsanalyse) nicht notwendigerweise einen Ausschluss ihrer Kausalität rechtfertigen.

Mutation c.783G>A [34]. Allele mit reduzierter Penetranz, die durch oft nicht bekannte genetische Faktoren (Modifier) beeinflusst wird, finden sich ebenfalls in den o.g. Datenbanken.

Die bei der Auswertung relevanten Datenbanken wie ExAC und gnomAD sollten als Populationskontrollen, aber keinesfalls als per se gesunde Kontrollen angesehen werden. Dies wird z.B. deutlich bei in ExAC/gnomAD erfassten Individuen, die Träger von Funktionsverlust-(Loss-of-Function, LoF)-Mutationen im ASXL1-Gen sind. ASXL1Keimbahnmutationen sind die Ursache des kongenitalen monogenen Bohring-Opitz-Syndroms [35]. Die in den Datenbanken identifizierten Individuen hingegen sind fast sämtlich ältere Personen, deren ASXL1-Mutationen sehr wahrscheinlich ausschließlich im
Blut vorhandene somatische Mutationen darstellen und mögliche Hinweise auf klonale Hämatopoese oder Leukämie sind [36]. Ähnliche Beispiele betreffen Mutationen in den Genen DNMT3A und TET2.

\section{Splicemutationen in der kodierenden} Sequenz: Nukleotidaustausche, die keine oder nur schwach wirksame Aminosäureaustausche $\mathrm{zu}$ verursachen scheinen, können eigentlich Splicemutationen sein [37]. Die Anwendung mehrerer leistungsfähiger Splice-Prädiktionsprogramme hilft vor allem, wenn der (erweiterte) Konsensus von Akzeptor- und Donor-Splicestellen betroffen ist oder solche Motive durch die Mutation neu entstehen (wie oft bei tief-intronischen Splicemutationen). Verlässliche Vorhersagen sind jedoch bioinformatisch kaum möglich, wenn exonische SpliceEnhancer-Motive betroffen sind. In der Aufdeckung solcher Mutationen liegt ein großes Potential der RNA-Sequenzierung [38-40]. Die systematische Anwendung von Transkriptom-Analysen für seltene Erkrankungen wird im Artikel von Prokisch behandelt.

Ein individueller WES- bzw. WGS-Datensatz kann 200-500 seltene („,private“) Varianten beinhalten: Dieser Herausforderung kann zukünftig besser durch die Hinzunahme weiterer „OmicsDaten“ des Patienten begegnet werden. Über RNA-/Transkriptom- hinaus bieten auch parallele Proteom-Analysen erste vielversprechende Ansätze [41, 42].

Bedeutung des Phänotyps: Genetische Varianten müssen immer im Zusam- 
menhang mit dem jeweiligen Phänotyp interpretiert werden. Die systematische Phänotypisierung und die Integration von Phänotyp-Ontologien in die Auswertung von WES-/WGS-Exom-Analysen war Thema des Artikels von Peter Krawitz, erschienen in Heft 1 der letzten MedGen-Ausgabe [43].

Auch die Varianteninterpretation für unzweifelhaft mit Erkrankungen assoziierte Gene ist nicht trivial, und das Vorkommen von Gain-of-Function- (GoF) und LoF-Mutationen im gleichen Gen können die Interpretation erschweren. Dies wird im Artikel durch Marschall et al. am Beispiel des LongQT-Syndroms erläutert.

\section{Zukunft der Genomik?}

Das Zeitalter der genomischen Medizin hat gerade erst begonnen. Die Implementation der Genomanalyse als StandardTest in der humangenetischen Diagnostik zeichnet sich ab bzw. ist in einigen europäischen Ländern (England, Frankreich, Niederlande) zunehmend Realität.

Gleichzeitig beschäftigen sich große Konsortien mit der Optimierung von WGS-/WES-Analysen (u. a. durch besseres Verständnis nicht-kodierender Mutationen, optimierte Bioinformatik, neueste genomische Technologien (z.B. Long-Read-Technologien, Epigenetik, Transkriptom-Analysen) oder durch Multi-Omics-Ansätze; z. B.: www.solverd.eu).

Mit der permanenten Weiterentwicklung im Bereich der Genomik und neuer Technologien ergeben sich ständig neue biologische Erkenntnisse und vielversprechende neue Ansätze in der Medizin:

- Long-Read-Sequenziertechnologien (ONT, PacBio) erlauben echte

Gesamtgenom-Analysen, evtl. de novo-Assemblierungen, des humanen Genoms.

- Long-Read-Mapping-Technologien (Bionano Genomics [https:// bionanogenomics.com/], Nabsys [http://nabsys.com/]) versprechen eine Revolutionierung oder sogar den Ersatz der Zytogenetik („next generation cytogenetics").
- Fast alle Genomik-Technologien werden auf Einzelzell-Niveau (singlecell) ermöglicht (single-cell RNAseq [44], single-cell ATACseq [45]). Diese Tendenz spiegelt sich u. a. auch im sogenannten Human Cell Atlas wider (https://www.humancellatlas.org/).

- „Spatial genomics“ oder in situSequenzierung (z. B. Nanostring (https://www.nanostring.com/), ReadCoor (https://www.readcoor. com/), Spatial Transcriptomics (https://spatialtranscriptomics. com/), Slideseq [46]) bieten die Chance, die klassische Histologie präziser zu machen und womöglich zu ersetzen.

- Es ist davon auszugehen, dass die heutigen Technologien weiter verbessert werden und der Preis pro Analyse weiter sinkt, u. a. weil neue Sequenzierer (z. B. MGIseq T7 [http://en. mgitech.cn] oder Genapsys [https:// www.genapsys.com/]) den Wettbewerb im Markt verschärfen werden.

Die stürmische Entwicklung der Genomik hat die wissenschaftlichen und diagnostischen Möglichkeiten der Humangenetik revolutioiniert - und tut dies weiterhin. Mit der zunehmenden Bedeutung für die gesamte Medizin und den einzelnen Patienten (u.a. hinsichtlich Gentherapien) wird die Rolle der Humangenetiker immer wichtiger.

\section{Korrespondenzadresse}

\section{Hanno J. Bolz}

Senckenberg Zentrum für Humangenetik

Frankfurt am Main, Deutschland

h.bolz@senckenberg-humangenetik.de

\section{Alexander Hoischen}

Department of Human Genetics \& Department of Internal Medicine, Radboud Institute of Medical Life Sciences, Radboud University Medical Center

Nijmegen, Niederlande

Alexander.Hoischen@radboudumc.nl

Interessenkonflikt. H.J. Bolz und A. Hoischen geben an, dass kein Interessenkonflikt besteht.

\section{Literatur}

1. Funari VA, Krakow D, Nevarez L et al (2010) BMPER mutation in diaphanospondylodysostosis identified by ancestral autozygosity mapping and targeted high-throughput sequencing. Am J Hum Genet 87(4):532-537

2. Rajadhyaksha AM, Elemento O, Puffenberger EG et al (2010) Mutations in FLVCR1 cause posterior column ataxia and retinitis pigmentosa. Am J Hum Genet 87(5):643-654

3. Najmabadi H, Hu H, Garshasbi M et al (2011) Deep sequencing reveals 50 novel genes for recessive cognitive disorders. Nature 478(7367):57-63

4. Ng SB, Turner EH, Robertson PD et al (2009) Targeted capture and massively parallel sequencing of 12 human exomes. Nature 461(7261):272-276

5. Hoischen A, van Bon BW, Gilissen C et al (2010) De novo mutations of SETBP1 cause Schinzel-Giedion syndrome. Nat Genet 42(6):483-485

6. de Ligt J, Willemsen $\mathrm{MH}$, van Bon BW et al (2012) Diagnostic exome sequencing in persons with severe intellectual disability. N Engl J Med 367(20):1921-1929

7. Rauch A, Wieczorek D, Graf E et al (2012) Range of genetic mutations associated with severe non-syndromic sporadic intellectual disability: an exome sequencing study. Lancet 380(9854):1674-1682

8. Vissers LE, de Ligt J, Gilissen C et al (2010) A de novo paradigm for mental retardation. Nat Genet 42(12):1109-1112

9. Lupski JR, Reid JG, Gonzaga-Jauregui C et al (2010) Whole-genome sequencing in a patient with Charcot-Marie-Tooth neuropathy. N Engl J Med 362(13):1181-1191

10. Sobreira NL, Cirulli ET, Avramopoulos D et al (2010) Whole-genome sequencing of a single proband together with linkage analysis identifies a Mendelian disease gene.Plos Genet 6(6):e1000991

11. Belkadi A, Bolze A, Itan Y et al (2015) Wholegenome sequencing is more powerful than wholeexome sequencing for detecting exome variants. Proc Natl Acad SciU SA 112(17):5473-5478

12. Gilissen C, Hehir-Kwa JY, Thung DT et al (2014) Genome sequencing identifies major causes of severe intellectual disability. Nature 511(7509):344-347

13. Wheeler DA, Srinivasan $M$, Egholm $M$ et al (2008) The complete genome of an individual by massively parallel DNA sequencing. Nature 452(7189):872-876

14. Ng SB, Buckingham KJ, Lee C et al (2010) Exome sequencing identifies the cause of a mendelian disorder. Nat Genet 42(1):30-35

15. Krawitz PM, Schweiger MR, Rodelsperger $C$ et al (2010) Identity-by-descent filtering of exome sequence data identifies PIGV mutations in hyperphosphatasia mental retardation syndrome. Nat Genet 42(10):827-829

16. Becker J, Semler O, Gilissen C et al (2011) Exome sequencing identifies truncating mutations in human SERPINF1 in autosomal-recessive osteogenesis imperfecta. Am J Hum Genet 88(3):362-371

17. Zheng GX, Lau BT, Schnall-Levin M et al (2016) Haplotyping germline and cancer genomes with high-throughput linked-read sequencing. Nat Biotechnol 34(3):303-311

18. Bohrson CL, Barton AR, Lodato MA et al (2019) Linked-readanalysis identifies mutations in singlecell DNA-sequencing data. Nat Genet. https://doi. org/10.1038/s41588-019-0366-2

19. Boycott KM, Rath A, Chong JX et al (2017) International cooperation to enable the diagnosis of all rare genetic diseases. Am J Hum Genet 100(5):695-705 
20. Wright CF, FitzPatrickDR, Firth HV (2018) Paediatric genomics: diagnosing rare disease in children. Nat Rev Genet 19(5):253-268

21. den Hollander Al, Koenekoop RK, Yzer S et al (2006) Mutations in the CEP290 (NPHP6) gene are a frequent cause of Leber congenital amaurosis. Am JHum Genet 79(3):556-561

22. Sangermano R, Garanto A, Khan M et al (2019) Deep-intronic $A B C A 4$ variants explain missing heritability in Stargardt disease and allow correction of splice defects by antisense oligonucleotides. Genet Med https://doi.org/10.1038/s41436-0180414-9

23. Bauwens M, Garanto A, Sangermano R et al (2019) ABCA4-associated disease as a model for missing heritability in autosomal recessive disorders: novel noncoding splice, cis-regulatory, structural, and recurrent hypomorphic variants. Genet Med https://doi.org/10.1038/s41436-018-0420-y

24. Johnston JJ, Williamson KA, Chou CM et al (2019) NAA10 polyadenylation signal variants cause syndromic microphthalmia. J Med Genet https:// doi.org/10.1136/jmedgenet-2018-105836

25. Lozano-Urena A, Ferron SR (2019) Beyond proteincoding genes. Elife 8 . https://doi.org/10.7554/ eLife. 45123

26. Ang CE, Ma Q, Wapinski OL et al (2019) The novel IncRNA Inc-NR2F1 is pro-neurogenic and mutated in human neurodevelopmental disorders. Elife 8. https://doi.org/10.7554/eLife.41770

27. Chaisson MJ, Huddleston J, Dennis MY et al (2015) Resolving the complexity of the human genome using single-molecule sequencing. Nature 517(7536):608-611

28. Jain M, Koren S, Miga KH et al (2018) Nanopore sequencing and assembly of a human genome with ultra-long reads. Nat Biotechnol 36(4):338-345

29. Joo JE, Dowty JG, Milne RL et al (2018) Heritable DNA methylation marks associated with susceptibility to breast cancer. Nat Commun 9(1):867

30. Freed D, Pevsner J (2016) The contribution of mosaic variants to autism spectrum disorder. Plos Genet 12(9):e1006245

31. Abu-Safieh L, Al-Anazi S, Al-Abdi L et al (2012) In search of triallelism in Bardet-Biedl syndrome. Eur J Hum Genet 20(4):420-427

32. Pauli S, Altmüller J, Schröder S et al (2018) Homozygosity for the c.428delG variant in KIAA0586 in a healthy individual: implications for molecular testing in patients with Joubert syndrome. J Med Genet https://doi.org/10.1136/ jmedgenet-2018-105470

33. Stephen LA, Tawamie H, Davis GM et al (2015) TALPID3 controls centrosome and cell polarity and the human ortholog KIAA0586 is mutated in Joubert syndrome (JBTS23). Elife 4. https://doi. org/10.7554/eLife.08077

34. Bessette AP, DeBenedictis MJ, Traboulsi El (2018) Clinical characteristics of recessive retinal degeneration due to mutations in the CDHR1 gene and a review of the literature. Ophthalmic Genet 39(1):51-55

35. Hoischen A, van Bon BW, Rodriguez-Santiago $B$ et al (2011) De novo nonsense mutations in ASXL1 cause Bohring-Opitz syndrome. Nat Genet 43(8):729-731

36. Carlston CM, O'Donnell-Luria AH, Underhill HR et al (2017) Pathogenic ASXL1 somatic variants in reference databases complicate germline variant interpretation for Bohring-Opitz Syndrome. Hum Mutat 38(5):517-523

37. Soukarieh O, Gaildrat P, Hamieh M et al (2016) Exonic splicing mutations are more prevalent than currently estimated and can be predicted by using in silico tools. Plos Genet 12(1):e1005756

38. Cummings BB, Marshall JL, Tukiainen T et al (2017) Improving genetic diagnosis in Mendelian disease with transcriptome sequencing. Sci Transl Med 9(386). https://doi.org/10.1126/scitranslmed. aal5209

39. Kremer LS, Bader DM, Mertes C et al (2017) Genetic diagnosis of Mendelian disorders via RNA sequencing. Nat Commun 8:15824

40. GonorazkyHD, NaumenkoS, Ramani AKetal(2019) Expanding the boundaries of RNA sequencing as a diagnostic tool for rare mendelian disease. Am J Hum Genet 104(3):466-483

41. Manzoni C, Kia DA, Vandrovcova J et al (2018) Genome, transcriptome and proteome: the rise of omics data and their integration in biomedical sciences. Brief Bioinformatics 19(2):286-302

42. Low TY, Mohtar MA, Ang MY, Jamal R (2018) Connecting proteomics to next-generation sequencing: proteogenomics and its current applications in biology. Proteomics. https://doi. org/10.1002/pmic.201800235

43. Krawitz P(2019) Klinisch-bioinformatische Analyse bei Intelligenzminderung. medgen 31:20. https:// doi.org/10.1007/s11825-019-0233-7

44. Cao J, Spielmann M, Qiu X et al (2019) The singlecell transcriptional landscape of mammalian organogenesis. Nature 566(7745):496-502

45. Buenrostro JD, Wu B, Litzenburger UM et al (2015) Single-cell chromatin accessibility reveals principles of regulatory variation. Nature 523(7561):486-490

46. Rodriques SG, StickelsRR, Goeva Aetal (2019) Slideseq: a scalable technology for measuring genomewide expression at high spatial resolution. bioRxiv. https://doi.org/10.1126/science.aaw1219

47. Nicolas G, Veltman JA (2019) The role of de novo mutations in adult-onset neurodegenerative disorders. Acta Neuropathol 137(2):183-207

48. Martin HC, Jones WD, Mclntyre R et al (2018) Quantifying the contribution of recessive coding variation to developmental disorders. Science 362(6419):1161-1164

49. Short PJ, McRae JF, Gallone G et al (2018) De novo mutations in regulatory elements in neurodevelopmental disorders. Nature 555(7698):611-616

50. Nilsson D, Pettersson M, Gustavsson P et al (2017) Whole-genome sequencing of cytogenetically balanced chromosome translocations identifies potentially pathological gene disruptions and highlights the importance of Microhomology in the mechanism of formation. Hum Mutat 38(2):180-192

51. Murcia Pienkowski V, Kucharczyk M, Mlynek M et al (2019) Mapping of breakpoints in balanced chromosomal translocations by shallow wholegenome sequencing points to EFNA5, BAHD1 and PPP2R5E as novel candidates for genes causing human Mendelian disorders. J Med Genet 56(2):104-112

52. Redin C, Brand H, Collins RL et al (2017) The genomic landscape of balanced cytogenetic abnormalities associated with human congenital anomalies. Nat Genet 49(1):36-45

53. Boycott KM, Innes AM (2017) When one diagnosis is not enough. NEngl J Med 376(1):83-85

54. Posey JE, Harel T, Liu P et al (2017) Resolution of disease phenotypes resulting from multilocus genomic variation. N Engl J Med 376(1):21-31

55. Ebermann I, Elsayed SM, Abdel-Ghaffar TY et al (2008) Double homozygosity for mutations of AGL and SCN9A mimicking neurohepatopathy syndrome. Neurology 70(24):2343-2344
56. Pfundt R, Del Rosario M, Vissers L et al (2017) Detection of clinically relevant copy-number variants by exome sequencing in a large cohort of genetic disorders. Genet Med 19(6):667-675

57. Wright CF, McRae JF, Clayton S et al (2018) Making new genetic diagnoses with old data: iterative reanalysis and reporting from genome-wide data in 1,133 families with developmental disorders. Genet Med 20(10):1216-1223

58. Biesecker LG, Spinner NB (2013) A genomic view of mosaicism and human disease. Nat Rev Genet 14(5):307-320

59. Lelieveld SH, Reijnders MR, Pfundt R et al (2016) Meta-analysis of 2,104 trios provides support for 10 new genes for intellectual disability. Nat Neurosci 19(9):1194-1196

60. Ercan-Sencicek AG, Jambi S, Franjic D et al (2015) Homozygous loss of DIAPH1 is a novel cause of microcephaly in humans. Eur J Hum Genet 23(2):165-172

61. Lynch ED, Lee MK, Morrow JE, Welcsh PL, Leon PE, King MC (1997) Nonsyndromic deafness DFNA1 associated with mutation of a human homolog of the Drosophila gene diaphanous. Science 278(5341):1315-1318

62. Platzer K, Cogne B, Hague J et al (2018) Haploinsufficiency of CUX1 causes nonsyndromic global developmental delay with possible catch-up development. Ann Neurol 84(2):200-207 